\title{
PCA3 Score and Prostate Cancer Diagnosis at Repeated Saturation Biopsy. Which cut-off: 20 or 35?
}

\author{
Pietro Pepe, Filippo Fraggetta, Antonio Galia, Giorgio Skonieczny, Francesco Aragona \\ Urology Unit, Cannizzaro Hospital (PP, FA), Pathology Unit, Cannizzaro Hospital (FF,AG) and \\ Department of Economy, University of Catania (GS), Catania, Italy
}

\section{ABSTRACT}

Purpose: To compare PCA3 score cut-off of 35 vs 20 in PCa diagnosis in patients undergoing repeated saturation prostate biopsy (SPBx).

Material and Methods: From January 2010 to May 2011, 118 patients (median 62.5 years) with primary negative extended biopsy underwent a transperineal SPBx (median 30 cores) for persistent suspicion of PCa. The indications for repeated biopsy were: persistently high or increasing PSA values; PSA > $10 \mathrm{ng} / \mathrm{mL}$, PSA values between 4.1-10 or 2.6-4 ng/mL with free/total PSA $\leq 25 \%$ and $\leq 20 \%$, respectively; moreover, before performing SPBx urinary PCA3 score was evaluated.

Results: All patients had negative DRE and median PSA was $8.5 \mathrm{ng} / \mathrm{mL}$ (range: $3.7-24$ $\mathrm{ng} / \mathrm{mL}$ ). A T1c PCa was found in 32 patients (27.1\%): PCA3 score was 59 (median; range: 7-201) in the presence of PCa and 35 (median; range: 3-253) in the absence of cancer $(\mathrm{p}<0.05)$. In the presence of ASAP and HGPIN median PCA3 score was 109 (range: 42-253) and 40 (range: 30-140), respectively. Diagnostic accuracy, sensitivity, specificity, PPV and NPV of PCA3 score cut-off of 20 vs 35 in PCa diagnosis were 44.9 vs $50 \%, 90.6$ vs $71.9 \%, 27.9$ vs $41.8 \%, 31.9$ vs $31.5 \%$ and 88.9 vs $80 \%$, respectively. ROC analysis demonstrated an AUC for PCA3 $\geq 20$ vs $\geq 35$ of 0.678 and 0.634 , respectively. Conclusions: Our data suggest that PCA3 is more useful as an exclusion tool; moreover, setting a PCA3 cut-off at 20 vs 35, would have avoided 22.9 vs $38.1 \%$ of biopsies while missing 9.4\% and 28\% diagnosis of PCa.

\section{ARTICLE INFO}

\section{Key words:}

PCA3; prostate cancer; diagnosis; biopsy

Int Braz J Urol. 2012; 38: 489-95

Submitted for publication:

November 30, 2011

Accepted after revision: June 11, 2012

\section{INTRODUCTION}

Widespread use of the PSA test along with the introduction of mass screening protocols in Europe (1) and in USA (2) have increased the detection rate of prostate cancer (PCa), currently the most prevalent malignancy in older men. Although the European Randomized Study of Screening for Prostate Cancer (ERSPC) reported a 20\% reduction of mortality in the screened arm with an estimated rate of over-diagnosis as high as $50 \%$ (1), $22-41 \%$ of men with persistent suspicion of PCa following negative primary biopsy were found to have cancer at repeated biopsy $(3,4)$. In an attempt to improve PSA specificity and reduce the number of unnecessary biopsies many molecular forms of PSA (i.e. free/total PSA, pro-PSA) have been brought into clinical practice, most notably in the presence of PSA values lower than $10 \mathrm{ng} / \mathrm{mL}$; in this light the Prostate Cancer Gene 3 (PCA3) assay (a gene-based marker) has recently been used to select patients for 
repeated prostate biopsy $(5,6)$. Many authors have reported a significant difference of PCA3 score in patients with positive or negative repeated biopsy $(7,8)$ underlining the lack of sensitivity of PCA3 to prostate volume and prostatitis (7); moreover, the PCA3 score has been incorporated into nomograms to assist in the decision to biopsy assignment of an individual risk of PCa (9).

Recent studies have demonstrated that a PCA3 score of 35 provides an optimal balance between sensitivity and specificity in diagnosing PCa with greater diagnostic accuracy than free/total PSA (cut-off 25\%) $(7,10)$; on the other hand, a PCA3 score cut-off lower than 25 could be predictive of pathological indolent PCa (pIPCa) (11).

Our scope is to report the accuracy of PCA3 cut-off of 35 vs 20 in PCa diagnosis in patients undergoing repeated saturation prostate biopsy (SPBx) for the persistence of suspicion of cancer.

\section{MATERIALS AND METHODS}

From January 2010 to May 2011, 118 consecutive cases, all of Caucasian origin and between the ages of 48 and 72 years (median 62.5 years) underwent a SPBx (median 30; range: 24-38 cores) 9 months (median; range 6-18) after a primary negative extended biopsy (median 18 cores). The 118 patients were selected from a case-finding protocol for PCa detection (12) and had one single previous negative biopsy. The indications for repeated biopsy were: persistently high or increasing PSA values; PSA > $10 \mathrm{ng} / \mathrm{mL}$, PSA values between 4.110 or $2.6-4 \mathrm{ng} / \mathrm{mL}$ with free/total PSA $\leq 25 \%$ and $\leq 20 \%$, respectively. SPBx was performed transperineally using a tru-cut 18 gauge needle (Bard; Covington, GA), a GE Logiq 500 PRO ecograph (General Electric; Milwaukee, WI) supplied with a biplanar transrectal probe (5-6.5 MHz) under sedation and antibiotic prophylaxis. The prostate biopsy protocol included a median of 12 cores in the posterior zone of each lobe (apex, median zone and base of the gland) beginning parasagittally to reach the outer edges of the gland (lateral margins) and 2-3 cores in the transition zone (4).

From three to ten days prior to performing SPBx, first-catch urine samples were collected following digital rectal examination (three strokes per lobe) and processed to quantify PCA3 and PSA mRNA concentrations using the PROGENSA PCA3 assay (Gen-Prob Inc. San Diego, CA USA); PCA3 score was calculated using the following equation: (PCA3 mRNA/PSA mRNA) x 1000 (13).

In the presence of $\mathrm{PCa}$ either definitive treatment or active surveillance (AS) was offered. Those desiring surgery underwent retropubic radical prostatectomy (RRP) with bilateral obturator and external iliac lymphadenectomy. The volume of cancer in the entire specimen was reported according to Bostwick (14). Each case was analyzed independently by two dedicated pathologists (FF,AG) and the incidence of PCa, that fulfilled the Epstein criteria (15) for pIPCa (cancer volume less than $0.5 \mathrm{~mL}$ and no Gleason grade 4 or 5 disease) was recorded.

The performance characteristics at different PCA3 score cut-off of 20 vs 35 were evaluated in terms of diagnostic accuracy, sensitivity, specificity, positive predictive value (PPV) and negative predictive value (NPV). Receiver operating characteristic (ROC) was performed to define diagnostic performance of PCA $3 \geq 20$ vs PCA $3 \geq 35$ using the calculated area under the curve (AUC); moreover, a probability (p) value of less than 0.05 was considered statistically significant.

\section{RESULTS}

All patients had negative DRE and median PSA was $8.5 \mathrm{ng} / \mathrm{mL}$ (range: 3.7-24 ng/mL): 50 (42.4\%) had PSA > $10 \mathrm{ng} / \mathrm{mL}, 65$ (55\%) had PSA between $4-10 \mathrm{ng} / \mathrm{mL}$ with PSA $\mathrm{F} / \mathrm{T} \leq 25 \%$ and 3 (2.6\%) had PSA between 2.6-4 ng/mL with PSA $\mathrm{F} / \mathrm{T} \leq 20 \%$, respectively. In all patients an adequate concentration of PCA3 and PSA mRNA was obtained; median PCA3 score was equal to 49 (range 3-253); 91 (77.1\%) and 73 (61.9\%) patients had a PCA3 score greater than 20 and 35, respectively.

A T1c PCa was found in 32 patients (27.1\%); median PSA was $12.5 \mathrm{ng} / \mathrm{mL}$ (range: $4.5-$ $24 \mathrm{ng} / \mathrm{mL}$ ): 15 and 17 men had a PSA > 10 and between 4-10 ng/mL, respectively. Gleason score (GS) was 6 in 26 cases (81.2\%), 7 in 4 (12.5\%) and 8 in 2 (6.3\%). Median positive cores were 2 (range: 1-14) with the presence of either only one positive 
core in 12 cases $(37.5 \%)$ or a microfocus of PCa (defined as a percentage of cancer $\leq 5 \%$ in a single core with $\mathrm{GS} \leq 6)$ in $6(19 \%)$; greatest percentage of cancer (GPC) was $\leq 50$ and $>50 \%$ in $22(68 \%)$ and $10(32 \%)$ cases, respectively. The remaining 73 men (61.8\%) had normal parenchyma, 4 (3.4\%) had ASAP and 9 (7.7\%) had HGPIN.

PCA3 score was 59 (median; range: 7-201) in the presence of $\mathrm{PCa}$ and 35 (median; range: $3-253)$ in the absence of cancer $(p<0.05)$; In the presence of ASAP and HGPIN median PCA3 score was 109 (range: 42-253) and 40 (range: 30-140), respectively.

PCa detection rate increased from 11.5\% with PCA3 score of less than 20 to $33.3 \%$ with scores greater than 100 (Figure-1); moreover, a PCA3 cut off of 20 and 35 found $29(90.6 \%)$ and 23 (71.9\%) PCa, respectively. Men with a PCA3 score $\geq 35$ had a $31.5 \%$ probability of a positive biopsy compared to $31.8 \%$ probability in patients with PCA3 score $\geq 20$. Median PCA3 score in the presence of GS equal to 6, 7 and 8 was 62, 68 and 145 , respectively. Setting a PCA3 cut-off at 20 vs 35 would have avoided $22.9 \%$ vs $38.1 \%$ of biopsies but $3(9.4 \%)$ and 9 cases (28\%) of cancer with GS of 6 would have been missed.
Diagnostic accuracy, sensitivity, specificity, PPV and NPV of PCA3 score cut-off of 20 vs 35 are listed in Table- 1 . ROC analysis demonstrated an AUC for PCA3 $\geq 20$ vs PCA3 $\geq 35$ of 0.678 and 0.634 , respectively.

Among the patients with $\mathrm{PCa} 4$ cases (12.5\%) underwent radiotherapy and 3 cases (10\%) followed AS protocol. None of the 25 patients submitted to RRP had a pIPCa (18 pT2cNo and 7 pT3aNo); the clinical (PSA, PSA F/T, PCA3 score) and pathological (biopsy and surgical specimens) characteristics are listed in Table-2.

\section{DISCUSSION}

Currently, with repeated prostate biopsy constituting $20-30 \%$ of all performed procedures, and low percentage discovery rates for PCa, certain clinical criteria for the selection of patients at high risk of developing PCa cancer are still lacking. Vlaeminck-Guillem (16) demonstrated that PCA3 predicts PCa multifocality while Ploussard (11) and Auprich (8) confirmed PCA3 to be a valuable predictor of pIPCa in selection between AS protocols or focal therapy. In the largest studies to date (1072, 463 and 226 cases) all undergoing

Figure 1 - PCa detection by PCA3 score range in the 118 patients submitted to repeated saturation biopsy.

No. of patients

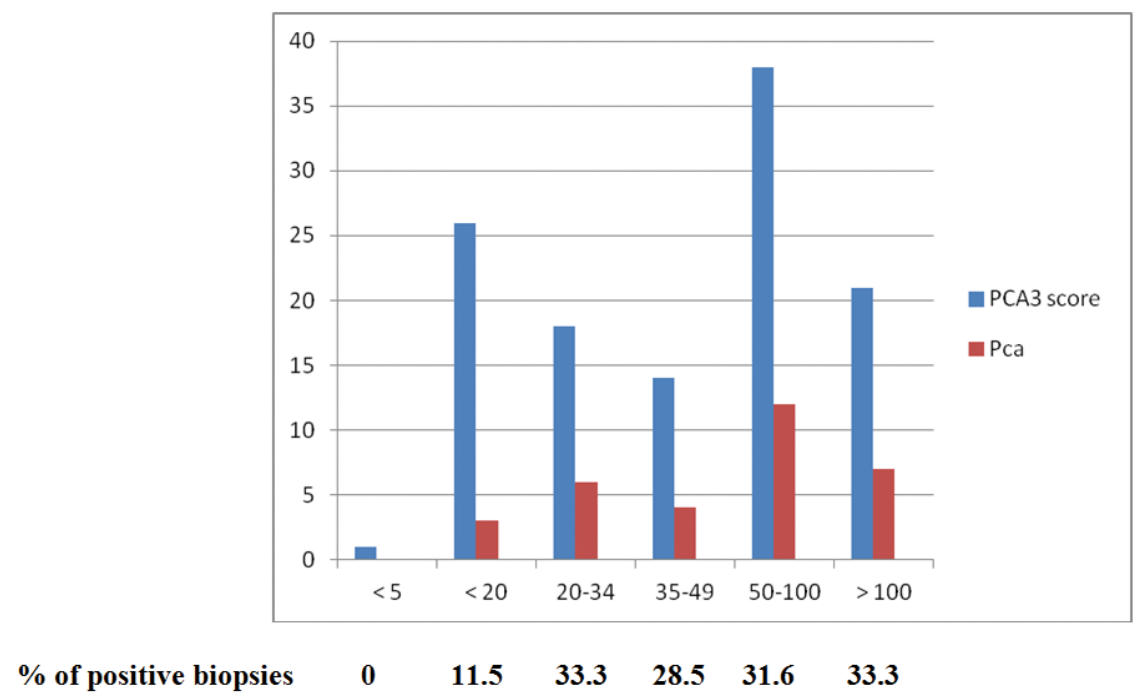


Table 1 - PCA3 score accuracy in diagnosing prostate cancer: cut-off 20 vs 35 .

\begin{tabular}{|c|c|c|c|}
\hline Accuracy & PCA3 $\geq 20$ & Vs & PCA3 $\geq 35$ \\
\hline Sensitivity & $90.6 \%$ & & $71.9 \%$ \\
\hline Specificity & $27.9 \%$ & & $41.8 \%$ \\
\hline PPV & $31.9 \%$ & & $31.5 \%$ \\
\hline NPV & $88.9 \%$ & & $80.0 \%$ \\
\hline Diagnostic accuracy & $44.9 \%$ & & $50.0 \%$ \\
\hline
\end{tabular}

$\mathbf{P P V}=$ positive predictive value; $\mathbf{N P V}=$ negative predictive value

repeated biopsy Aubin (10), Haese (7) and Marks (5) respectively reported positive biopsy probability [upon comparison of PCA3 score < 5-10 to PCA3 score $=100$ ] as being 6 vs $12 \%, 12$ vs $57 \%$ and 47 vs 50\%. Aubin (10) demonstrated that a PCA3 cut-off equal to 35 predicts biopsy outcomes 2 years in advance resulting more accurate than PSA; moreover, Haese (7) at a PCA3 cut-off of 20 vs 35 reported a $44 \%$ vs $67 \%$ reduction of repeated biopsies missing 12 vs $21 \%$ of cancers with $\mathrm{GS} \geq 7$.

Despite these interesting reports, PCA3 accuracy (cut-off $\geq 35$ ) at repeated biopsy remains contradictory: sensitivity, specificity, PPV and NPV range between 47 to $76.6 \%$ (5), 66.6 to $78.6 \%$ (6), 39 to $74 \%$ (16) and 62.5 to $87 \%$ (17), respectively; in fact, only NPV is satisfactory being $>80 \%$ in three of the five studies evaluated $(5,6,17,18,19)$.

Recently, Roobol (20) reported that PCA3 cannot replace the PSA test as the choice of an appropriate cut-off level with acceptable performance is debatable and Rigau (21) suggested the usefulness of a multiplexed urine-based diagnostic test having the same sensitivity as the PSA test.

In our series, the best parameter was NPV equal to $88.9 \%$ and $80 \%$ using a cut-off of 20 and 35 , respectively; to the contrary PPV resulted the worst (about 31\%) due to the high number of false positive results. These data suggest that PCA3 is more useful as an exclusion tool; moreover, a PCA3 cut-off of 20 in comparison with PSA demonstrates a good sensitivity (90.6\%) reducing the number of unnecessary repeated biopsy (22.9\% of the cases) and minimizes the risk of missing significant PCa (3 out 32 cases).

Regarding our results three further considerations need to be made. Firstly, we don't know if the false positive results correlate to SPBx false negatives or, on the other hand, could be predictive for future PCa detection (22): 4 men had an HGPIN and 8 an ASAP; moreover, 11 (12.8\%) out of 86 patients with negative SPBX had a PCA3 score greater than 100 that allows a risk to detect a cancer at repeated biopsy equal to 30\% (23). Secondly, due to the limited number of cases submitted to RRP (25 patients) the accuracy of PCA3 score in diagnosing pIPCa cannot be established, especially in patients with a PCA3 score below 20; however, overall pIPCa detection rate is lower in case-finding protocols $(7.7 \%$ of the patients in case of repeated SPBX) (6) in comparison with screening programs for $\mathrm{PCa}$ (50\% of the cases) (1). Finally, detection rate for PCa after repeated SPBX $(27.1 \%)$ resulted similar to that observed with standard repeated biopsy protocol (27\% for Marks and 28\% for Haese, respectively) $(5,6)$; probably, the performance of a 18 cores biopsy protocol as initial procedure decreased detection rate for PCa at repeated SPBX.

\section{CONCLUSIONS}

PCA3 score should be considered to be a complementary parameter in the clinical management of repeated biopsy candidates; in our pre- 
Table 2 - Clinical and pathological characteristics of the 25 patients with prostate cancer submitted to retropubic radical prostatectomy.

\begin{tabular}{|c|c|c|c|c|c|c|c|c|c|c|}
\hline $\begin{array}{l}\text { age } \\
\text { (yrs) }\end{array}$ & $\begin{array}{c}\mathrm{PSA} \\
\mathrm{ng} / \mathrm{mL}\end{array}$ & $\begin{array}{c}\text { biopsy } \\
\text { GS }\end{array}$ & $\begin{array}{l}\text { positive } \\
\text { cores }\end{array}$ & $\begin{array}{c}\text { GPC } \\
\%\end{array}$ & $\begin{array}{c}\text { PSA } \\
\text { F/T \% }\end{array}$ & $\begin{array}{l}\text { PCA3 } \\
\text { score }\end{array}$ & pTN & $\begin{array}{c}\text { definitive } \\
\text { GS }\end{array}$ & psm & $\begin{array}{c}\mathrm{PCa} \\
\text { volume }\end{array}$ \\
\hline 60 & 4.5 & $3+3$ & 1 & 50 & 10 & 35 & T2c No & $3+3$ & - & $>0.5 \mathrm{~mL}$ \\
\hline 62 & 8 & $3+3$ & 1 & 50 & 8 & 37 & T2c No & $3+3$ & - & $>0.5 \mathrm{~mL}$ \\
\hline 72 & 6.8 & $3+3$ & 1 & 50 & 10 & 86 & T2c No & $3+3$ & - & $>0.5 \mathrm{~mL}$ \\
\hline 70 & 9.9 & $3+3$ & 1 & 50 & 15 & 17 & T2b N0 & $3+3$ & - & $>0.5 \mathrm{~mL}$ \\
\hline 58 & 8.9 & $3+3$ & 1 & 60 & 12 & 119 & T2c No & $3+3$ & - & $>0.5 \mathrm{~mL}$ \\
\hline 63 & 9.6 & $3+3$ & 1 & 50 & 11 & 54 & T2a NO & $3+3$ & - & $>0.5 \mathrm{~mL}$ \\
\hline 62 & 9.2 & $3+3$ & 1 & 75 & 12 & 74 & T2c No & $3+3$ & + & $>0.5 \mathrm{~mL}$ \\
\hline 69 & 9.5 & $3+3$ & 1 & 50 & 9 & 32 & T2c NO & $3+3$ & - & $>0.5 \mathrm{~mL}$ \\
\hline 70 & 9.5 & $3+3$ & 1 & 50 & 8 & 40 & T3a NO & $3+3$ & - & $>0.5 \mathrm{~mL}$ \\
\hline 64 & 7.9 & $3+3$ & 2 & 50 & 9 & 73 & T2c No & $3+3$ & - & $>0.5 \mathrm{~mL}$ \\
\hline 58 & 6.9 & $3+3$ & 1 & 5 & 23 & 41 & T2c No & $3+3$ & - & $>0.5 \mathrm{~mL}$ \\
\hline 68 & 8.3 & $3+3$ & 1 & 5 & 11 & 8 & T2a NO & $3+3$ & - & $>0.5 \mathrm{~mL}$ \\
\hline 65 & 8.3 & $3+3$ & 1 & 5 & 27 & 30 & T2a NO & $3+3$ & - & $>0.5 \mathrm{~mL}$ \\
\hline 63 & 10 & $3+3$ & 1 & 5 & 20 & 79 & T2c No & $3+3$ & - & $>0.5 \mathrm{~mL}$ \\
\hline 64 & 13.5 & $3+3$ & 1 & 5 & 11 & 170 & T3a NO & $3+3$ & - & $>0.5 \mathrm{~mL}$ \\
\hline 65 & 11.5 & $3+3$ & 1 & 50 & 11 & 58 & T2c NO & $3+3$ & - & $>0.5 \mathrm{~mL}$ \\
\hline 68 & 12.8 & $3+3$ & 4 & 100 & 15 & 30 & T3a N0 & $3+3$ & - & $>0.5 \mathrm{~mL}$ \\
\hline 60 & 8.8 & $3+3$ & 3 & 75 & 23 & 110 & T2c No & $3+3$ & + & $>0.5 \mathrm{~mL}$ \\
\hline 70 & 10.8 & $3+3$ & 3 & 50 & 19 & 48 & T2c No & $3+4$ & - & $>0.5 \mathrm{~mL}$ \\
\hline 67 & 11.5 & $3+3$ & 8 & 50 & 13 & 45 & T2c No & $3+4$ & - & $>0.5 \mathrm{~mL}$ \\
\hline 69 & 12 & $3+4$ & 9 & 100 & 18 & 62 & T3a NO & $4+3$ & + & $>0.5 \mathrm{~mL}$ \\
\hline 61 & 10.8 & $3+4$ & 6 & 75 & 19 & 47 & T3a NO & $4+3$ & + & $>0.5 \mathrm{~mL}$ \\
\hline 61 & 24 & $4+4$ & 5 & 100 & 19 & 73 & T3a N0 & $4+4$ & + & $>0.5 \mathrm{~mL}$ \\
\hline 62 & 10.5 & $3+4$ & 6 & 75 & 8 & 74 & T2c No & $3+4$ & - & $>0.5 \mathrm{~mL}$ \\
\hline 61 & 12.5 & $3+4$ & 3 & 100 & 8 & 79 & T3a N0 & $4+4$ & + & $>0.5 \mathrm{~mL}$ \\
\hline
\end{tabular}

GS = Gleason score; $\mathbf{G P C}=$ greatest percentage of cancer; $\mathbf{P S A} \mathbf{F} / \mathbf{T}=$ free/total; $\mathbf{p s m}=$ positive surgical margins . 
liminary dataset, the high NPV equal to 88.9 (cut off of 20) and 80\% (cut off of 35) should limit the use of PCA3 score to avoid the risk of unnecessary repeated biopsy. Moreover, setting a PCA3 cut-off at 20 vs 35 , would have avoided 22.9 vs $38.1 \%$ of biopsies while missing 3 (9.4\%) and 9 cases (28\%) of significant PCa, respectively.

\section{ABBREVIATIONS}

$\mathrm{PCa}=$ prostate cancer;

PCA3 = Prostate Cancer Gene 3;

pIPCa $=$ pathological indolent prostate cancer;

$\mathrm{SPBx}=$ saturation prostate biopsy;

$\mathrm{PPV}=$ positive predictive value;

NPV = negative predictive value;

$\mathrm{GS}=$ Gleason score;

$\mathrm{GPC}=$ greatest percentage of cancer;

PSA $\mathrm{F} / \mathrm{T}$ = free/total PSA;

psm $=$ positive surgical margins;

$\mathrm{ROC}=$ receiver operating characteristic;

$\mathrm{AUC}=$ area under the curve.

\section{CONFLICT OF INTEREST}

None declared.

\section{REFERENCES}

1. Schröder FH, Hugosson J, Roobol MJ, Tammela TL, Ciatto $\mathrm{S}$, Nelen V, et al.: Screening and prostate-cancer mortality in a randomized European study. N Engl J Med. 2009; 360: 1320-8.

2. Andriole GL, Crawford ED, Grubb RL 3rd, Buys SS, Chia $D$, Church TR, et al.: Mortality results from a randomized prostate-cancer screening trial. N Engl J Med. 2009; 360: 1310-9. Erratum in: N Engl J Med. 2009; 360: 1797.

3. Walz J, Graefen M, Chun FK, Erbersdobler A, Haese A, Steuber $\mathrm{T}$, et al.: High incidence of prostate cancer detected by saturation biopsy after previous negative biopsy series. Eur Urol. 2006; 50: 498-505.

4. Pepe P, Aragona F: Saturation prostate needle biopsy and prostate cancer detection at initial and repeat evaluation. Urology. 2007; 70: 1131-5.

5. Marks LS, Fradet Y, Deras IL, Blase A, Mathis J, Aubin SM, et al.: PCA3 molecular urine assay for prostate cancer in men undergoing repeat biopsy. Urology. 2007; 69: 532-5.
6. Pepe P, Aragona F: PCA3 score vs PSA free/total accuracy in prostate cancer diagnosis at repeat saturation biopsy. Anticancer Res. 2011; 31: 4445-9.

7. Haese A, de la Taille A, van Poppel H, Marberger M, Stenzl A, Mulders PF, et al.: Clinical utility of the PCA3 urine assay in European men scheduled for repeat biopsy. Eur Urol. 2008; 54: 1081-8.

8. Auprich M, Chun FK, Ward JF, Pummer K, Babaian R, Augustin $\mathrm{H}$, et al.: Critical assessment of preoperative urinary prostate cancer antigen 3 on the accuracy of prostate cancer staging. Eur Urol. 2011; 59: 96-105.

9. Perdonà $\mathrm{S}$, Cavadas V, Di Lorenzo G, Damiano R, Chiappetta $G$, Del Prete P, et al.: Prostate cancer detection in the "grey area" of prostate-specific antigen below $10 \mathrm{ng} / \mathrm{mL}$ : head-tohead comparison of the updated PCPT calculator and Chun's nomogram, two risk estimators incorporating prostate cancer antigen 3. Eur Urol. 2011; 59: 81-7.

10. Aubin SM, Reid J, Sarno MJ, Blase A, Aussie J, Rittenhouse $\mathrm{H}$, et al.: PCA3 molecular urine test for predicting repeat prostate biopsy outcome in populations at risk: validation in the placebo arm of the dutasteride REDUCE trial. J Urol. 2010; 184: 1947-52.

11. Ploussard G, Durand X, Xylinas E, Moutereau S, Radulescu $C$, Forgue $\mathrm{A}$, et al.: Prostate cancer antigen 3 score accurately predicts tumour volume and might help in selecting prostate cancer patients for active surveillance. Eur Urol. 2011; 59: 422-9.

12. Pepe $P$, Aragona $F$ : Incidence of insignificant prostate cancer using free/total PSA: results of a case-finding protocol on 14,453 patients. Prostate Cancer Prostatic Dis. 2010; 13: 316-9.

13. Groskopf J, Aubin SM, Deras IL, Blase A, Bodrug S, Clark C, et al.: APTIMA PCA3 molecular urine test: development of a method to aid in the diagnosis of prostate cancer. Clin Chem. 2006; 52: 1089-95.

14. Bostwick DG, Grignon DJ, Hammond ME, Amin MB, Cohen $M$, Crawford $D$, et al.: Prognostic factors in prostate cancer. College of American Pathologists Consensus Statement 1999. Arch Pathol Lab Med. 2000; 124: 995-1000.

15. Epstein JI, Walsh PC, Carmichael M, Brendler CB: Pathologic and clinical findings to predict tumor extent of nonpalpable (stage T1c) prostate cancer. JAMA. 1994; 271: 368-74.

16. Vlaeminck-Guillem V, Devonec M, Colombel M, RodriguezLafrasse C, Decaussin-Petrucci M, Ruffion A: Urinary PCA3 score predicts prostate cancer multifocality. J Urol. 2011; 185: 1234-9.

17. Fradet $Y$, Saad F, Aprikian A, Dessureault J, Elhilali M, Trudel $C$, et al.: UPM3, a new molecular urine test for the detection of prostate cancer. Urology. 2004; 64: 311-5; discussion 315-6.

18. Schilling D, Hennenlotter J, Munz M, Bökeler U, Sievert KD, Stenzl A: Interpretation of the prostate cancer gene 3 in reference to the individual clinical background: implications for daily practice. Urol Int. 2010; 85: 159-65. 
19. Vlaeminck-Guillem V, Ruffion A, André J, Devonec M, Paparel P: Urinary prostate cancer 3 test: toward the age of reason? Urology. 2010; 75: 447-53.

20. Roobol MJ: Contemporary role of prostate cancer gene 3 in the management of prostate cancer. Curr Opin Urol. 2011; 21: $225-9$.

21. Rigau M, Morote J, Mir MC, Ballesteros C, Ortega I, Sanchez $A$, et al.: PSGR and PCA3 as biomarkers for the detection of prostate cancer in urine. Prostate. 2010; 70: 1760-7.

22. Remzi M, Haese A, Van Poppel H, De La Taille A, Stenzl A, Hennenlotter J, et al.: Follow-up of men with an elevated PCA3 score and a negative biopsy: does an elevated PCA3 score indeed predict the presence of prostate cancer? BJU Int. 2010; 106: 1138-42.
23. Roobol MJ, Schröder FH, van Leenders GL, Hessels D, van den Bergh RC, Wolters T, et al.: Performance of prostate cancer antigen 3 (PCA3) and prostate-specific antigen in Prescreened men: reproducibility and detection characteristics for prostate cancer patients with high PCA3 scores $(\geq 100)$. Eur Urol. 2010; 58: 893-9.
Correspondence address: Dr. Pietro Pepe Urology Unit - Cannizzaro Hospital Via Messina 829, Catania, Italy Fax: + 3995 726-3259 E-mail: piepepe@hotmail.com 